\title{
Abordagem intrabucal para exérese de sialolito na glândula sublingual: relato de caso
}

Intrabucal approach to exeresis of sublingual gland sialolith - case report

Abordaje intraoral de la escisión del sialolito en la glándula sublingual: reporte de caso

\section{Carla Cecília Lira Pereira de CASTRO ${ }^{1}$}

Éwerton Daniel Rocha RODRIGUES ${ }^{2}$

Belmiro Cavalcanti do Egito VASCONCELOS ${ }^{2}$

Thaís Cristina Araújo MOREIRA ${ }^{3}$

${ }^{1}$ Faculdade de Odontologia - FOP/UPE, Universidade de Pernambuco - UPE, 50100-130 Recife - PE, Brasil

${ }^{2}$ Departamento de Cirurgia e Traumatologia Bucomaxilofacial, Hospital Universitário Oswaldo Cruz - HUOC, Universidade de Pernambuco - UPE 50100-130 Recife-PE, Brasil

${ }^{3}$ Departamento de Cirurgia e Traumatologia Bucomaxilofacial, Hospital Universitário da Universidade Federal do Piauí, Universidade Federal do Piauí - UFPI, 64049-550 Ininga - PI, Brasil

\section{Resumo}

A sialolitíase é uma patologia que afeta as glândulas salivares e tem como patogênese a obstrução dos ductos dessas glândulas, causando uma diminuição do fluxo salivar. O local de acometimento depende da anatomia e de fatores fisiológicos inerentes a cada glândula. O presente estudo tem como objetivo relatar um caso de sialolitíase da glândula sublingual, evidenciando diagnóstico e terapêutica. O relato de caso trata-se de paciente do gênero feminino, 53 anos de idade, melanoderma, apresentou-se ao ambulatório do serviço de Cirurgia e Traumatologia Buco-Maxilo-Facial do Hospital Universitário da Universidade Federal do Piauí, queixando-se de sensação álgica e desconforto associado ao aumento de volume na região sublingual, com sintomatologia aumentada durante as refeições. No exame extrabucal não se observaram alterações. Entretanto, no exame intrabucal podia ser observado um aumento de volume endurecido e consistente à palpação, associado a desconforto. Ao exame radiográfico foi observado uma área radiopaca, circunscrita e com limites bem definidos, sugestiva de sialolito. O tratamento proposto foi a excisão cirúrgica sob anestesia local. Foi realizado bloqueio do nervo lingual e anestesia infiltrativa no soalho bucal (lidocaína 1:100.00 com epinefrina) e incisão póstero-anterior após identificação da área, seguida de dissecção romba. Após a remoção do cálculo, foi realizada a sutura com vicryl 4-0. A paciente foi acompanhada durante 09 meses e observou-se reparo tecidual satisfatório, remissão da sintomatologia e a função normal da glândula. Conclui-se que a abordagem de tratamento depende do tamanho e da localização do sialolito, sendo sempre preferível métodos minimamente invasivos.

Descritores: Glândula Sublingual; Cálculos dos Ductos Salivares; Cálculos das Glândulas Salivares; Diagnóstico Bucal.

\section{Abstract}

Sialolithiasis is a pathology that affects the salivary glands and its pathogenesis is the obstruction of the ducts of these glands, causing a decrease in salivary flow. The site of involvement depends on the anatomy and physiological factors inherent to each gland. The present study aims to report a case of sialolithiasis of the sublingual gland, showing diagnosis and therapy. The case report is a female patient, 53 years old, melanoderma, presented to the outpatient clinic of the Buco-Maxillofacial Surgery and Traumatology service of the University Hospital of the Federal University of Piauí, complaining of sensation pain and discomfort associated with swelling in the sublingual region, with increased symptoms during meals. In the facial examination, no changes were observed. However, in the intraoral examination, an increase in hardened volume consistent with palpation could be observed, associated with discomfort. Upon radiographic examination, a radiopaque area was observed, circumscribed and with well-defined limits, suggestive of sialolith. The proposed treatment was surgical excision under local anesthesia. Lingual nerve block and infiltrative anesthesia were performed on the oral floor (lidocaine 1: 100.00 with epinephrine) and a posteroanterior incision after identification of the area, followed by blunt dissection. After removing the stone, suture was performed with 4-0 vicryl. The patient was followed for 9 months and satisfactory tissue repair, remission of symptoms and normal gland function were observed. It is concluded that the treatment approach depends on the size and location of the sialolith, with minimally invasive methods always preferable.

Descriptors: Sublingual Gland; Salivary Duct Calculi; Salivary Gland Calculi; Diagnosis, Oral.

\section{Resumen}

La sialolitiasis es una patología que afecta a las glándulas salivales y su patogenia es la obstrucción de los conductos de estas glándulas, provocando una disminución del flujo salival. El sitio de afectación depende de la anatomía y factores fisiológicos inherentes a cada glándula. El presente estudio tiene como objetivo reportar un caso de sialolitiasis de la glándula sublingual, mostrando diagnóstico y terapia. El caso clínico es una paciente de 53 años, melanoderma, que acudió a la consulta externa del servicio de Cirugía Buco-Maxilofacial y Traumatología del Hospital Universitario de la Universidad Federal de Piauí, quejándose de sensación. dolor y malestar asociados con hinchazón en la región sublingual, con aumento de los síntomas durante las comidas. En el examen del arnés, no se observaron cambios. Sin embargo, en el examen intraoral se pudo observar un aumento del volumen endurecido acorde con la palpación, asociado a malestar. Al examen radiográfico se observó una zona radiopaca, circunscrita y con límites bien definidos, sugerente de sialolito. El tratamiento propuesto fue la exéresis quirúrgica bajo anestesia local. Se realizó bloqueo del nervio lingual y anestesia infiltrativa en el piso oral (lidocaína 1: 100.00 con epinefrina) e incisión posteroanterior después de la identificación de la zona, seguida de disección roma. Tras retirar el cálculo, se realizó la sutura con vicryl 4-0. Se siguió al paciente durante 9 meses y se observó una reparación tisular satisfactoria, remisión de los síntomas y una función glandular normal. Se concluye que el abordaje del tratamiento depende del tamaño y la ubicación del sialolito, siendo siempre preferibles los métodos mínimamente invasivos.

Descriptores: Glándula Sublingual; Cálculos del Conducto Salival; Cálculo de las Glándulas Salivales; Diagnóstico Bucal.

INTRODUÇÃo

A sialolitíase é uma patologia que afeta as glândulas salivares e tem como patogênese a obstrução dos ductos dessas glândulas, causando uma diminuição do fluxo salivar. Cerca de 80 a $90 \%$ dos sialolitos são encontrados na glândula submandibular, 5-20\% na glândula parótida e a menor parte dos casos na glândula sublingual e nas glândulas salivares menores, que são raramente afetadas ${ }^{1,2}$. O acometimento da glândula sublingual por esta patologia está associado a traumas mecânicos e conseguinte formação de cálculos, resultado da mineralização de detritos que se acumulam no ducto glandular ${ }^{1,3}$. 
O edema, acompanhado ou não de dor durante as refeições, é o principal sintoma dessa desordem, podendo acometer tanto o sítio esquerdo quanto 0 direito, sendo 0 acometimento bilateral raro ${ }^{2}$. Além disso, infecções repetidas e formação de abscessos dolorosos também podem estar presentes ${ }^{1,4,5}$. $\mathrm{Na}$ maioria dos estudos, relata-se uma predominância pelo sexo masculino e por pacientes entre a 3-6 décadas de vida. Seu acometimento em crianças é raro, mas pode afetar qualquer idade ${ }^{1}$.

Os sialolitos das glândulas sublinguais são geralmente redondos ou ovais e, na maioria dos casos, cerca de 70 a $80 \%$ dos pacientes apresentam apenas 1 sialolito, 20\% apresentam 2 e apenas $5 \%$ dos pacientes apresentam 3 ou mais. Estes podem apresentar superfície lisa ou áspera, podendo variar de um grão pequeno a um gigante sialolito, chegando a $2 / 3 \mathrm{~cm}$ de diâmetro ${ }^{1,2}$. A composição salivar dos pacientes pode ser um indicio da formação do cálculo, uma vez que o aumento de cálcio salivar pode estar envolvido na etiopatologia da doença $a^{3,5}$.

Esta patologia é geralmente diagnosticada em radiografias oclusais de rotina, geralmente como uma área radiopaca, entretanto a composição do cálculo pode influenciar sua radiopacidade ${ }^{2}$. Quando se localizam nos ductos glandulares, 0 comprometimento do fluxo salivar é mais acentuado do que quando se localizam no interior da glândula6. O tratamento varia de acordo com a localização do cálculo. Quando acomete a glândula sublingual, consiste em uma abordagem cirúrgica através da sialadenectomia completa, através de um acesso transoral ${ }^{1}$.

O objetivo do artigo foi relatar um caso de sialolitíase na glândula sublingual, trazendo uma breve revisão sistemática sobre o tema e correlacionando com a etiopatologia e condutas terapêuticas realizadas no presente caso.

\section{CASO CLÍNICO}

Paciente do sexo feminino, 53 anos de idade, melanoderma, apresentou-se ao ambulatório do serviço de Cirurgia e Traumatologia Bucomaxiilofacial do HU-UFPI queixando-se de sensação álgica e desconforto associado a aumento de volume na região sublingual. A mesma relatou que a sintomatologia aumentava de intensidade durante as refeições e desapareciam em um curto período de tempo, não mais que 3 horas. A paciente vinha sofrendo com esses sintomas há cerca de 11 dias e não relatou outra sintomatologia ou doenças sistêmicas. No exame extrabucal não se observaram alterações significativas no contorno facial. No exame intrabucal a paciente relatou desconforto à palpação da região sublingual, onde podia ser observado um discreto aumento de volume endurecido e consistente à palpação (Figura $1 \mathrm{~A})$. Foram solicitadas radiografias panorâmica e oclusal, onde foi possível observar uma área radiopaca, circunscrita, de limites bem definidos, sendo a principal hipótese diagnóstica um sialólito (Figura 1B).

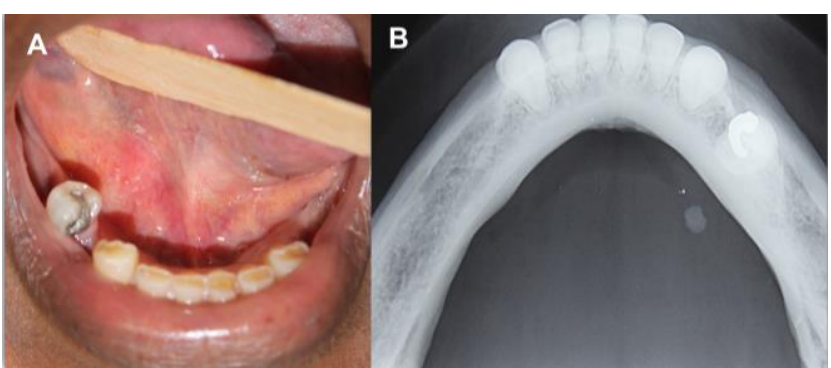

Figura 1: A) Aspecto intra-bucal pré-operatório. B) Radiografia oclusal evidenciando área radiopaca em região sublingual.

O tratamento proposto foi a excisão cirúrgica sob anestesia local. Foi realizada bloqueio do nervo lingual e anestesia infiltrativa no soalho bucal (lidocaína 1:100.00 com epinefrina). Após identificação da área endurecida através da palpação foi realizada uma incisão póstero-anterior, seguida de dissecção romba (Figura 2A).

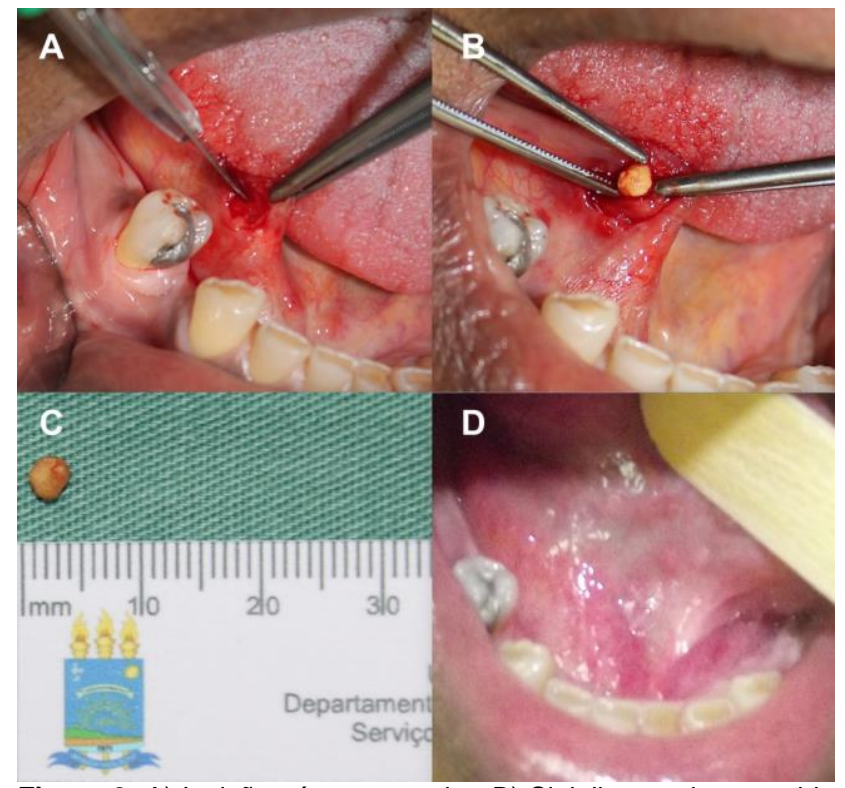

Figura 2: A) Incisão póstero-anterior. B) Sialolito sendo removido. C) Cálculo salivar medindo aproximadamente $5 \mathrm{~mm}$ em seu maior diâmetro. D) Aspecto pós-operatório de 30 dias.

Um cálculo salivar medindo cerca de $4 \mathrm{~mm}$ foi removido (Figura $2 \mathrm{~B}$ e $2 \mathrm{C}$ ) e, em seguida, foi realizado a sutura com vicryl 4-0. Para o controle da dor e da inflamação pósoperatória foram prescritos lbuprofeno $300 \mathrm{mg}$ e paracetamol $750 \mathrm{mg}$, a paciente foi orientada a realizar higiene da cavidade oral com o auxílio de colutório (clorexidina $0,12 \%$ duas vezes ao dia) e a manter a dieta rica em líquidos, 
proteínas e alimentos cítricos. Nas consultas pós-operatórias, foi possível observar reparo tecidual satisfatório, remissão da sintomatologia e a função normal da glândula sublingual. A paciente encontra-se em 09 meses de pósoperatório e permanece sem queixas (Figura 2D).

DISCUSSÃO

Quando se compara o sistema da glândula submandibular com o da parótida e da sublingual, encontram-se alguns fatores fisiológicos que justificam sua maior susceptibilidade à esta desordem. Lustmann et al. $^{2}$ e Hong et al. ${ }^{1}$ acreditam que alguns fatores anatômicos e fisiológicos favoreçam o aparecimento na glândula submandibular, como a maior concentração de cálcio, $\mathrm{o} \mathrm{pH}$ mais alcalino, o trajeto do ducto de Wharton longo e tortuoso, e a própria ação da gravidade dificulta o fluxo salivar, facilitando a sua obstrução ${ }^{1,2}$. Por outro lado, o acometimento da glândula sublingual é raro e está associado a traumas ${ }^{1}$. A paciente do caso aqui apresentado não relatou traumas na região.

O acometimento dos sítios direito e esquerdo são equivalentes, entretanto é baixa a incidência bilateral. Segundo Pizzirani et al. ${ }^{7}$ é maior o número de casos se dá no sitio esquerdo, concordando com os achados do presente caso. Não há predileção por sexo ou idade, embora a maioria dos estudos relate um maior acometimento em indivíduos do sexo masculino entre a 3-6 décadas de vida ${ }^{1}$. Concordando com este fato, Kawakami et al. $^{8}$ afirmaram que a sialolitíase ocorre preferencialmente em homens, entre 30 a 40 anos de idade. No entanto, Castro ${ }^{9}$ afirma a predileção pelo sexo feminino e o acometimento de indivíduos entre 41 e 60 anos idade, corroborando com os achados do presente relato.

Na maioria dos casos, os cálculos não ultrapassam 10 milímetros de diâmetro (88\%) sendo apenas $7,6 \%$ maiores que 15 milímetros. Estes são denominados de cálculos salivares glandulares gigantes (GGSC), considerado raros6. Geralmente encontram-se em forma redonda ou ovalada quando se trata da glândula sublingual ${ }^{1}$. Esta desordem pode ser sintomática ou não, dependendo do tamanho do cálculo, podendo apresentar edema doloroso, agravado durante as refeições devido ao aumento da secreção salivar e conseguinte pressão intraglandular ${ }^{1,6}$. O caso descrito neste artigo se enquadra nas características que acontecem na maioria dos casos.

O tamanho e a localização do sialolito são determinantes para a gravidade da doença. Quando localizados no interior do ducto glandular, o comprometimento do fluxo salivar é mais acentuado. No interior do ducto, o cálculo salivar tem forma fusiforme, cilíndrica ou esférica, podendo obstruir o escoamento salivar de forma parcial ou total. Quando intraglandulares, os cálculos são aglomerados irregulares e multifacetados, necessitando-se na maioria dos casos realizar a exérese da glândula ${ }^{6}$.

O exame radiográfico serve para auxiliar o diagnóstico. Entretanto, alguns cálculos não são visíveis em radiograficamente. O que pode ser explicado, possivelmente, pelo grau de mineralização dos cálculos ${ }^{2,6}$. Segundo o estudo de Lustmann et al. ${ }^{2}$, a calcificação dos sialolitos interfere na sua radiopacidade, o que faz com que possam ser identificados (ou não) em exames radiográficos. Aproximadamente $80 \%$ dos sialólitos são radiopacos, enquanto os outros $20 \%$ apresentam-se radiolúcidos ${ }^{2,10}$. No caso em tela, foi possível observar a presença de uma massa radiopaca no exame radiográfico oclusal, o que facilitou a excisão cirúrgica da patologia. Segundo Kawata et al. ${ }^{10}$, quando os sialólitos são diagnosticados em radiografia panorâmica de rotina, em que a imagem sobrepõe a mandíbula, é importante realizar uma radiografia oclusal para excluir possibilidade de outras lesões radiopacas. Da mesma forma, realizou-se no presente caso.

A escolha do tratamento também deve ser em função do tamanho e posição do sialolito. Quando não se consegue remover o cálculo através de uma terapia conservadora com compressas mornas, massagem em combinação com o uso de sialogogos e estimulo do fluxo salivar, opta-se por uma abordagem invasiva que pode consistir em litotripsia extracorpórea, sialendoscopia e sialoadenectomia ${ }^{5,6}$. A litotripsia extracorpórea caracteriza-se pela fragmentação do cálculo por ultrassom ou ondas de laser, quando estes são maiores que $4 \mathrm{~mm}$ de diâmetro, enquanto os menores destinam-se a sialendoscopia. Esta localiza e remove o cálculo usando uma câmera e uma sonda de cesta ${ }^{5}$. O estudo de Nahleili et al. ${ }^{11}$ avaliou a eficiência e segurança da utilização da sialendoscopia como única abordagem de tratamento e obteve sucesso em $80 \%$ dos casos. Iro et al. $^{12}$ foi o primeiro a descrever a lilotripsia, a qual atua destruindo o cálculo por fragmentação e permitindo a evacuação com saliva. Embora seja uma boa alternativa sem complicações, ela necessita de uma máquina especifica e de alto custo ${ }^{4}$.

A remoção transoral é o tratamento de 
escolha em pacientes com cálculos que podem ser palpados bimanualmente e foi descrita por Benazzou et al. ${ }^{13} \mathrm{e}$ indicada para cálculos com diâmetro superior a $8 \mathrm{~mm}$, como uma alternativa à sialendoscopia ${ }^{4}$. Os cálculos situados em uma região distal podem ser localizados por via transoral, devido sua a localização anterior. Entretanto, quando estes se localizam no istmo glandular ou em uma região proximal dentro do ducto um acesso extrabucal e até mesmo a sialoadenectomia podem ser indicados ${ }^{1,2,10}$. Tendo em vista a localização superficial do cálculo do presente caso, foi realizada a excisão cirúrgica e sialoadenectomia concordando com a literatura.

CONCLUSÃO

O uso de métodos minimamente invasivos para o tratamento de sialolitíase deve ser a primeira linha de tratamento. Uma abordagem intrabucal é um método rápido, com baixos custos e com alta taxa de sucesso. Esse método pode ser recomendado para remoção de cálculos localizados na região distal e superificial dos ductos salivares com baixas taxa de complicações pós-operatórias.

\section{REFERÊNCIAS}

1. Hong $\mathrm{KH}$, Yang YS. Sialolithiasis in the sublingual gland. J Laryngol Otol. 2003;117(11):905-7.

2. Lustmann J, Regev E, Melamed Y. Sialolithiasis on 245 patients and a review of the literature. Int J Oral Maxillofac Surg. 1990;19:135-38

3. Su Y, Zhang K, Ke Z, Zheng G, Chu M, Liao G. Increased calcium and decreased magnesium and citrate concentrations of submandibular/sublingual saliva in sialolithiasis. Arch Oral Biol. 2010;55(1):15-20.

4. Ruiz R, Brygo A, Nicot R, Ferri J. Sialolithiasis removal under general anesthesia: $A$ descriptive retrospective study in the maxillofacial surgery department in Lille University Hospital. J Stomatol Oral Maxillofac Surg. 2018;119(2):97-101.

5. Duong LT, Kakiche T, Ferré F, Nawrocki L, Bouattour A. Management of anterior submandibular sialolithiasis. J Oral Med Oral Surg. 2019;25(2):16.

6. Starling CR, Silva DT, Falcão AFP. Sialolitíase em glândula sublingual: relato de caso clínico. R Ci med biol. 2012;11(3):346-50.

7. Pizzirani C, Capuano A, Gemesio B, Simondi R. Considerazioni clinico-statistiche su 102 casi di calcolosi salivare. Mondo Odontostomatol. 1985:27:41-51.

8. Kawakami RY, Padovan LEM, Matsumoto MA, Ribeiro Júnior PD, Fraga SC. Sialolitíase recorrente: relato de caso clínico. Rev Fac Odontol Lins. 1997:10(1):33-9.
9. Castro AL. Estomatologia. 2.ed. São Paulo: Santos; 1995.

10. Kawata LT, Baldan RCF, Moraes NP, Biazolla ER. Sialolitíase em ducto de glâandula submandibular: relato de dois casos clínicos. Rev Odontol Araçatuba. 2006;27(1):14-18.

11. Nahlieli O, Baruchin AM. Sialoendoscopy: three years' experience as a diagnostic and treatment modality. J Oral Maxillofac Surg. 1997;55:912

12. Iro $\mathrm{H}$, Zenk J. Laser lithotripsy of salivary duct stones. Adv Otorhinolaryngol.1995;49:148-52.

13. Benazzou S, Salles F, Chenet F, Chossegros C. Exérèse des lithiases postérieures de la glande submandibulaire par abord endobuccal Transoral removal of submandibular hilar calculi. Rev Stomatol Chir Maxillofac. 2008; 109:163-66.

\section{CONFLITO DE INTERESSES}

Os autores declaram não haver conflitos de interesse

\section{AUTOR PARA CORRESPONDÊNCIA}

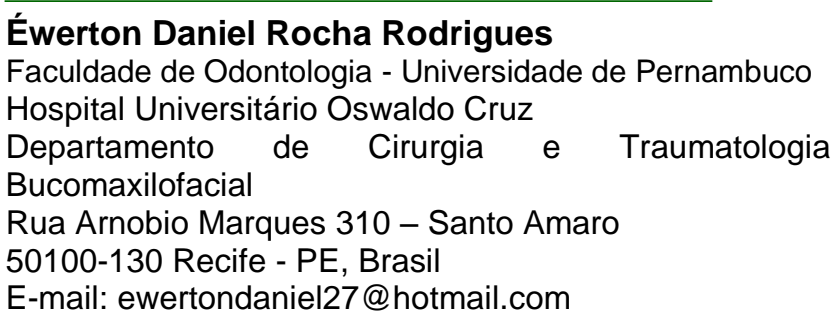

Submetido em 07/09/2020 Aceito em 23/10/2020 